\title{
What Drives Osman El Atwani Forward in Nuclear Materials Research
}

\author{
Kaitlin Calva
}

In the April 2019 issue of $J O M$, Osman El Atwani and his co-authors provided insights into the performance of nanocrystalline materials under extremes as part of the technical topic, Deformation and Transitions at Grain Boundaries. Their paper, "Interplay Between Grain Boundaries and Radiation Damage," describes both limitations of current studies and also suggests future works to overcome those limitations.

"My current research focuses on developing nanocrystalline and ultrafine metallic materials and investigating their performance under irradiation and mechanical extremes," said El Atwani of his work at Los Alamos National Laboratory (LANL). He started out at LANL as a Director's Postdoctoral Fellow in 2016, adding the G.T. Seaborg Postdoctoral Fellowship in 2017. The following year he received a Postdoctoral Distinguished Performance Award before beginning his current position as scientist 2 (Materials Science and Technology Division, MST8) in January 2019. "It is probably the most exciting time in my career as I was introduced to more challenges in working in different materials," he reflected. "This step was not going to be possible without the tremendous help of Stuart Maloy and other mentors I have worked with before."

The mentors El Atwani has worked with have made a great impact on his career, as he noted that his work in nuclear materials began with the help of his Ph.D. advisor at Purdue University, Jean Paul Allain (currently at the University of Illinois at Urbana-Champaign). As a materials engineering student, El Atwani was interested in this area, but it was not until a discussion with Allain that really pushed him to take the next step and get involved. "My advisor was very eager to work in this area and we were determined to make an impact in this field. He made the field look very exciting to me. We started with building our facility, Particle and Radiation
Interaction of Hard and Soft Matter (PRIHSM), to characterize materials with multi-tools in-situ." He recalled that, "it was a tiresome but fun process where I learned how to proceed and advance in this field."

Looking to the future, El Atwani talked of building on his achievements and his current work in the areas of nuclear power and understanding phenomena at the nanoscale/ to move the field forward. "Advancing nuclear power through next generation fission reactors and fusion devices is very exciting. I hope to see the first nuclear fusion power plant."

In addition to guidance from his mentors, El Atwani acknowledged the role that his membership and engagement in TMS played in helping to advance his career goals. "I met most of my collaborators through TMS. I was introduced to LANL scientists at TMS, which led me to apply to LANL and start my career," El Atwani said. "The TMS annual meeting is a place to communicate and discuss results, meet scientists and engineers, and move forward with collaborations and future plans."

The lessons El Atwani has learned throughout his career also make for good advice to others seeking a career in research. "This field requires a lot of brainstorming, learning, and planning," he said. "Identify your goals and spend time to find the pathway for success. This means you have to recognize the tools you need to master and the collaborators who can complement your experience. Collaboration is as important as the experience of the researcher, and TMS is one of the best places to start this process."

And when it comes to publishing results, he advises to not just look at a journal's impact factor, but to also find a journal where your work can make its own mark. "Try to impact the society with your work," he said. "While other people's judgments can be important and affect you, remember that your own judgment is what drives you forward." 